\title{
Equilibrium partitioning of macromolecules in confining geometries: Improved universality with a new molecular size parameter
}

Wang, Yanwei; Peters, Günther H.J.; Hansen, Flemming Yssing; Hassager, Ole

Published in:

Journal of Chemical Physics

Link to article, DOI:

$10.1063 / 1.2842073$

Publication date:

2008

Document Version

Publisher's PDF, also known as Version of record

Link back to DTU Orbit

Citation (APA):

Wang, Y., Peters, G. H. J., Hansen, F. Y., \& Hassager, O. (2008). Equilibrium partitioning of macromolecules in confining geometries: Improved universality with a new molecular size parameter. Journal of Chemical Physics, 128(12), [124904]. https://doi.org/10.1063/1.2842073

\section{General rights}

Copyright and moral rights for the publications made accessible in the public portal are retained by the authors and/or other copyright owners and it is a condition of accessing publications that users recognise and abide by the legal requirements associated with these rights.

- Users may download and print one copy of any publication from the public portal for the purpose of private study or research.

- You may not further distribute the material or use it for any profit-making activity or commercial gain

- You may freely distribute the URL identifying the publication in the public portal 


\title{
Equilibrium partitioning of macromolecules in confining geometries: Improved universality with a new molecular size parameter
}

\author{
Yanwei Wang, ${ }^{1, a)}$ Günther H. Peters, ${ }^{2, b)}$ Flemming Y. Hansen, ${ }^{3, c)}$ and Ole Hassager ${ }^{1, d)}$ \\ ${ }^{1}$ Danish Polymer Center, Department of Chemical Engineering, Technical University of Denmark, DK-2800 \\ Kgs. Lyngby, Denmark \\ ${ }^{2}$ MEMPHYS-Center for Biomembrane Physics, Department of Chemistry, Technical University of Denmark, \\ DK-2800 Kgs. Lyngby, Denmark \\ ${ }^{3}$ Department of Chemistry, Technical University of Denmark, DK-2800 Kgs. Lyngby, Denmark
}

(Received 11 December 2007; accepted 17 January 2008; published online 27 March 2008)

\begin{abstract}
We present a new framework for the description of macromolecules subject to confining geometries. The two main ingredients are a new computational method and the definition of a new molecular size parameter. The computational method, hereafter referred to the confinement analysis from bulk structures (CABS), allows the computation of equilibrium partition coefficients as a function of confinement size solely based on a single sampling of the configuration space of a macromolecule in bulk. Superior in computational speed to previous computational methods, CABS is capable of handling slits, channels, and box confining geometries for all molecular architectures. The new molecular size parameter, hereafter referred to the steric exclusion radius $R_{s}$, is explicitly defined and computed for a number of rigid objects and flexible polymers. We suggest that $R_{s}$ is the relevant molecular size parameter for characterization of spatial confinement effects on macromolecules. Results for the equilibrium partition coefficient in the weak confinement regime depend only on the ratio of $R_{S}$ to the confinement size regardless of molecular details. (C) 2008 American Institute of Physics. [DOI: 10.1063/1.2842073]
\end{abstract}

\section{INTRODUCTION}

The effect of a confining geometry on polymer properties has been a field of interest for more than 40 years, ${ }^{1}$ and the outcome of these studies has not only revealed most essential physical concepts of confined polymer systems, ${ }^{2-4}$ but has also found applications in areas such as polymer chromatography, surface lubrication, colloidal stabilization, and recently developed micro-/nanofluidic systems. ${ }^{5-13}$ For example, various chromatographic and membrane separation processes such as size exclusion chromatography (SEC) are governed by the partitioning of macromolecules between an unconfined bulk solution and a solution in a microscopic confining geometry. The partition phenomenon is described by the partition coefficient (pore-to-bulk concentration ratio) $K=c_{I} / c_{E}$, where $c_{I}$ and $c_{E}$ are the number densities of macromolecules inside the confining geometry and in bulk solution, respectively. In the case of purely steric exclusion where enthalpic effect is neglected, the equilibrium partition coefficient $K_{0}$ is determined only by the change in configurational entropy $\Delta S$ as the molecule is brought into the confining geometry from the bulk solution ${ }^{5}$ and is given by $K_{0}$ $=\exp \left(\Delta S / k_{B}\right)$, where $k_{B}$ is the Boltzmann constant. Without exception, $\Delta S<0$ and therefore $K_{0}<1$.

Several theoretical approaches have been employed to determine $K_{0}$ as a function of the molecule-to-pore size ratio. Analytical expressions have been obtained for a number of

\footnotetext{
${ }^{a)}$ Electronic mail: wyw@kt.dtu.dk.

${ }^{b)}$ Electronic mail: ghp@kemi.dtu.dk.

${ }^{c)}$ Electronic mail: flemming@kemi.dtu.dk.

d)Electronic mail: oh@kt.dtu.dk.
}

rigid molecules that only have orientational degree of freedom. ${ }^{14}$ For flexible polymers, Casassa ${ }^{1}$ derived expressions for $K_{0}$ by solving the diffusion equation for a linear random walk (RW) chain in a number of different confining geometries. This approach was later extended to symmetric star polymers by Casassa and Tagami ${ }^{15}$ and to asymmetric star polymers, pom-pom, and comb polymers confined in a slit confining geometry by Teraoka. ${ }^{16}$ Those concise and elegant results are important but are strictly valid only in the long chain limit. ${ }^{17} \mathrm{~A}$ fundamental understanding of the partitioning behavior of macromolecules in confining geometries can be obtained by the scaling theory. ${ }^{2}$ Daoud and de Gennes ${ }^{18}$ employed a blob picture to describe a confined linear self-avoiding walk (SAW) chain and predicted that the equilibrium partition coefficient follows $\ln K_{0} \sim-N(b / d)^{5 / 3}$, where $N$ is the total number of chain segments, $b$ is the segment length, and $d$ is the confinement size. The scaling exponent $5 / 3$ was verified by Wang and Teraoka ${ }^{19}$ using Monte Carlo simulations. The treatment of Daoud and de Gennes was later extended to a linear RW chain where $\ln K_{0} \sim-N(b / d)^{2}{ }^{20}$ The scaling concept provides a physical understanding of the effect of spatial confinement. However, it is not quantitative, and the results are strictly valid only in the high confinement regime where $\sqrt{N} b / d \gg 1$.

Monte Carlo (MC) studies of the equilibrium partitioning of macromolecules in confining geometries were initiated by Dayantis and Sturm. ${ }^{21}$ They discussed the statistical consequences of using either absorbing or reflecting boundary conditions in MC simulations and verified Casassa's results with an absorbing boundary. Davidson et al. ${ }^{17}$ observed by off-lattice MC simulations that $K_{0}$ is not only a function of 
the molecule-to-pore size ratio $R_{g} / d$ where $R_{g}$ is the radius of gyration of the macromolecule, but is also a function of either the segment-to-pore size ratio $b / d$ or the total number of chain segments $N$. Simulation results for a linear RW chain approach Casassa's solution for $b \rightarrow 0$ or equivalently for $N$ $\rightarrow \infty$. Their findings demonstrated the effect of chain flexibility on the equilibrium partitioning behavior, i.e., $K_{0}$ of a semiflexible chain can be substantially higher than that of a flexible chain with the same $R_{g} / d$. MC studies of the partitioning of linear excluded volume polymers and branched polymers in well defined confining geometries enable us to quantify the effect of solvent quality and chain architecture on the partitioning behavior. Cifra et al. ${ }^{22}$ and Bleha et al. ${ }^{23}$ showed by lattice MC simulations that a SAW chain can enter the confining geometry more easily than a RW chain of the same $R_{g}$. Chen and Escobedo ${ }^{8}$ investigated the effect of molecular topology (star, dendrimer, and hyperbranched polymers) on the properties of isolated polymer chains trapped in a slit with repulsive walls. The polymer linear dimension, density profiles across the slit, and confinement force were obtained, and the equilibrium partition coefficient was then estimated from the confinement force versus the slit width. A twin box method was also developed ${ }^{24,25}$ to study the equilibrium partitioning of concentrated solutions, which facilitates the investigation of concentration effect on $K_{0}$. In these MC studies of the partitioning of macromolecules, the confinement size $d$ needs to be explicitly specified for each simulation, ${ }^{8-10,17,19,21-23,26-31}$ and thus after sampling the orientational and conformational degrees of freedom of the studied macromolecule, only a single point is obtained on a plot of $K_{0}$ as a function of molecule-to-pore size ratio, such as $R_{g} / d$, as apposed to the new method that we are going to describe below.

The purpose of most prior calculations of the equilibrium partition coefficient as a function of the molecule-topore size ratio is to understand the separation principle of SEC. ${ }^{14-16,32,33}$ It is widely accepted that SEC separates polymer molecules according to their sizes in dilute solutions, but which size parameter to use has been a matter of debate for nearly 40 years. ${ }^{34,35}$ In theoretical studies of the separation principle of SEC, it is generally accepted that the separation is controlled by equilibrium thermodynamics ${ }^{36}$ and is based on an equilibrium partitioning of macromolecules between the mobile phase and the internal pore volumes due to steric exclusion effect. ${ }^{1,14}$ Therefore, efforts have been put to find the most relevant molecular size parameter that characterizes the equilibrium partitioning behavior. It was first found by Casassa and Tagami ${ }^{15}$ that the plots of $K_{0}$ as a function of $R_{g} / d$ do not follow a common curve for a linear RW polymer and star polymers of different number of arms. In an effort to bring these plots into a universal relationship, they proposed using $\left(R_{g} / d\right) g^{-1 / 3}$, where $g$ is the Zimm-Stockmayer branching parameter, ${ }^{37}$ which is defined as the ratio between the mean-square radius of gyration of a branched polymer and a linear polymer of the same molecular weight. More recently, Radke $^{32,33}$ and Teraoka ${ }^{16}$ proposed the use of the hydrodynamic radius $R_{h}$. Both $\left(R_{g} / d\right) g^{-1 / 3}$ and $R_{h}$ are capable of providing better universality than the use of $R_{g}$. However, the success was limited, and a fundamental understanding of the partitioning behavior is still lacking.

In the present work, a novel computational method is developed, which allows the computation of $K_{0}$ as a function of confinement size after only one sampling of the configuration space of a macromolecule in bulk. This method is hereafter referred to as a confinement analysis from bulk structures (CABS) method. Its origin is from a simple geometric consideration of the chain conformation and is based on a statistical physics formulation of a single chain under spatial confinement. For the computation of $K_{0}$, CABS is capable of handling slits, channels, and box confining geometries and is directly applicable to the study of branched polymers. Our method is computational efficient and also naturally leads us to introduce a new molecular size parameter which gives a better universal relationship between $K_{0}$ and confinement size. This molecular size parameter, hereafter referred to the steric exclusion radius $R_{S}$, is explicitly defined and can be determined easily from molecular simulations. We suggest that $R_{s}$ is the relevant molecular length scale for characterization of spatial confinement effects on macromolecules. In particular, for slit, channel, and box confining geometries, the equilibrium partition coefficients $K_{0}$ in the weak confinement regime depend only on the ratio of $R_{s}$ to the confinement size $d$ regardless of molecular details.

The paper is organized as follows. In Sec. II, the CABS method and the steric exclusion radius $R_{s}$ are introduced based on a statistical physics formulation of the equilibrium partitioning of polymers in the dilute solution limit. In Sec. III, we provide a brief description of the numerical details regarding the implementation of our CABS method and the calculation of $R_{s}$. Results are presented in Sec. IV along with discussions regarding some properties of this new molecular size parameter $R_{s}$, numerical validations of the CABS method, and the observation of a universal partitioning behavior when $K_{0}$ is shown as a function of $R_{s} / d$. Finally, a summary is given in Sec. V.

\section{THEORY}

\section{A. A grand canonical ensemble formulation}

We follow the statistical physics formulation of equilibrium partitioning of solvated polymer chains in the interior of a porous medium by Teraoka. ${ }^{5}$ When a porous medium with an inert surface is immersed into a solution of polymers, solvent molecules will fill the pore channels, and some polymer chains will migrate into the pore channels. Eventually, a concentration equilibrium is established between the interior of the porous medium and the exterior solution. It is convenient to treat the solution system in the grand canonical ensemble because both the interior and exterior (bulk) solutions are open systems. For simplicity, we regard each monomer as a united atom and denote by $\{\mathbf{r}\}_{i} \equiv\left\{\mathbf{r}_{1}, \mathbf{r}_{2}, \ldots, \mathbf{r}_{N}\right\}_{i}$ the collection of monomer position vectors of the $i$ th polymer chain where the suffix $i$ runs over all polymer chains. The configuration part of the Hamiltonian $H_{n}$ of the $n$ chain system is written as 


$$
H_{n}\left(\{\mathbf{r}\}_{1}, \ldots,\{\mathbf{r}\}_{n}\right)=\sum_{i=1}^{n} U_{1}\left(\{\mathbf{r}\}_{i}\right)+\sum_{i<j}^{n} U_{2}\left(\{\mathbf{r}\}_{i},\{\mathbf{r}\}_{j}\right),
$$

where $U_{1}\left(\{\mathbf{r}\}_{i}\right)$ includes both the intramolecular potential and molecule-wall interaction for the $i$ th chain with configuration $\{\mathbf{r}\}_{i}$, and $U_{2}\left(\{\mathbf{r}\}_{i},\{\mathbf{r}\}_{j}\right)$ is the interaction between the $i$ th and $j$ th chain, which is assumed to be pairwise additive. We do not consider solvent molecules explicitly and therefore omit solute-solvent interaction terms in the Hamiltonian. The conformation of a single polymer chain in $\Theta$-solvent condition is described by RW, while its conformation in good solvent condition is described by SAW. ${ }^{38}$ Therefore, the difference in the partitioning behavior of a RW chain and an SAW chain reflects the solvent effect on the partitioning behavior of macromolecules. In other words, the solvent effect is considered implicitly by modifying the form of $U_{1}$ and $U_{2}$. The grand canonical partition function is given by ${ }^{39}$

$$
\Xi(V, T, \mu)=\sum_{n=0}^{\infty} Q(n, V, T) \lambda^{n},
$$

where $\lambda=\exp (\beta \mu)$ is an absolute activity, $\beta=1 /\left(k_{B} T\right)$, and $\mu$ is the chemical potential of the polymer. The classical partition function for interacting particles $Q(n, V, T)$ is given by

$$
Q(n, V, T)=\frac{1}{n !}\left(\frac{1}{\Lambda}\right)^{3 n \times N} Z_{n \times N},
$$

where $n \times N$ is the total number of monomers in the system. $\Lambda=\sqrt{h^{2} /\left(2 \pi m k_{B} T\right)}$ is the De Broglie thermal wavelength, stemming from the integration over a Maxwell distribution of momenta for monomers of mass $m$, and $h$ is Planck's constant. $Z_{n \times N}$ is the configuration integral for $n$ polymer chains with $N$ monomers on each chain and is given by

$$
Z_{n \times N}=\int \cdots \int \exp \left[-\beta H_{n}\right] d\{\mathbf{r}\}_{1} \ldots d\{\mathbf{r}\}_{n} .
$$

Integration with respect to $\{\mathbf{r}\}_{i}$ is carried out for all configurations of the $i$ th chain. The concentration $c=\langle n\rangle / V$, where $\langle\cdots\rangle$ denotes an ensemble average, is calculated by

$$
c=\frac{1}{V} \frac{\partial \ln \Xi}{\partial \ln \lambda},
$$

separately for the exterior and the interior solutions, but with the same $\lambda$.

When the concentration is low so that binary interactions are negligible $\left(U_{2}=0\right)$, Eq. (4) reduces to $Z_{n \times N}=Z_{1 \times N}^{n}$, where $Z_{1 \times N}$ is the configuration integral of a single chain with $N$ monomers and is given by

$$
Z_{1 \times N}=\int \exp \left[-\beta U_{1}(\{\mathbf{r}\})\right] d\{\mathbf{r}\} .
$$

Under this condition, Eq. (2) reduces to

$$
\Xi=\exp \left(\lambda \Lambda^{-3 N} Z_{1 \times N}\right),
$$

and the concentration can simply be determined from Eq. (5) as

$$
c=\lambda \Lambda^{-3 N} Z_{1 \times N} V^{-1} .
$$

Since $\lambda$ and $\Lambda$ are common to both exterior and interior solutions, the equilibrium partition coefficient $K_{0}$ in the dilute solution limit is given by

$$
K_{0}=\frac{Z_{1 \times N, I} / V_{I}}{Z_{1 \times N, E} / V_{E}}
$$

where $V_{E}$ and $V_{I}$ are the volumes of the exterior and the interior solution systems, respectively. The partition functions $Z_{1 \times N, E}$ and $Z_{1 \times N, I}$ are calculated for a chain of $N$ monomers in the exterior and the interior solutions, respectively.

The variable of integration in Eq. (6) can be decomposed into $\mathbf{r}_{1}$ and $\{\mathbf{R}\}$, where $\mathbf{R}_{l} \equiv \mathbf{r}_{l}-\mathbf{r}_{1}(l=2, \ldots, N)$ denotes the configuration local to each chain. For the exterior solution, the single chain potential $U_{1 E}$ depends only on the internal variables, and $\int d \mathbf{r}_{1}=V_{E}$. We therefore obtain

$$
Z_{1 \times N, E} / V_{E}=\int \exp \left[-\beta \epsilon_{M}(\{\mathbf{R}\})\right] d\{\mathbf{R}\},
$$

with $U_{1 E}(\{\mathbf{r}\}) \equiv \epsilon_{M}(\{\mathbf{R}\})$. For solutions inside the porous media, there are two contributions to $U_{1 I}(\{\mathbf{r}\})$, i.e., an intramolecular interaction term $\epsilon_{M}(\{\mathbf{R}\})$ which depends only on the internal variables, and the interactions between the macromolecule and the pore walls $\epsilon_{M p}\left(\{\mathbf{R}\}, \mathbf{r}_{1}\right)$. We have

$$
U_{1 I}(\{\mathbf{r}\}) \equiv \epsilon_{M}(\{\mathbf{R}\})+\epsilon_{M p}\left(\{\mathbf{R}\}, \mathbf{r}_{1}\right) .
$$

Therefore,

$$
\begin{aligned}
Z_{1 \times N, I} / V_{I} & =\frac{1}{V_{I}} \int d\{\mathbf{r}\} \exp \left[-\beta U_{1 I}(\{\mathbf{r}\})\right] \\
& =\int q(\Omega,\{\mathbf{R}\}) \exp \left[-\beta \epsilon_{M}(\{\mathbf{R}\})\right] d\{\mathbf{R}\},
\end{aligned}
$$

where $q(\Omega,\{\mathbf{R}\})$ depends both on the confining geometry $\Omega$ and the polymer internal configuration and is given by

$$
q(\Omega,\{\mathbf{R}\})=\frac{1}{V_{I}} \int_{V_{I}} \phi\left(\Omega,\{\mathbf{R}\}, \mathbf{r}_{1}\right) d \mathbf{r}_{1},
$$

with $\phi\left(\Omega,\{\mathbf{R}\}, \mathbf{r}_{1}\right)$ defined as

$$
\phi\left(\Omega,\{\mathbf{R}\}, \mathbf{r}_{1}\right)=\exp \left[-\beta \epsilon_{M p}\left(\{\mathbf{R}\}, \mathbf{r}_{1}\right)\right] .
$$

Substitution of Eqs. (10) and (12) into Eq. (9) leads to

$$
\begin{aligned}
K_{0} & =\frac{\int q(\Omega,\{\mathbf{R}\}) \exp \left[-\beta \epsilon_{M}(\{\mathbf{R}\})\right] d\{\mathbf{R}\}}{\int \exp \left[-\beta \epsilon_{M}(\{\mathbf{R}\})\right] d\{\mathbf{R}\}} \\
& \equiv\langle q(\Omega,\{\mathbf{R}\})\rangle .
\end{aligned}
$$

The ensemble average $\langle\cdots\rangle$ denotes an average over the orientational and conformational freedom of the macromolecule. We focus here only on the steric exclusion effect that restricts the conformational entropy available to an individual polymer chain. Therefore, in the absence of specific surface interactions other than purely steric exclusion by solid walls, $\epsilon_{M p}$ is either 0 or $\infty$. Consequently, the Boltzmann factor $\phi\left(\Omega,\{\mathbf{R}\}, \mathbf{r}_{1}\right)$ is 1 for all molecular configurations with the first chain segment located at $\mathbf{r}_{1}$ free from wall overlap and 0 for overlapping configurations. $q(\Omega,\{\mathbf{R}\})$ can 


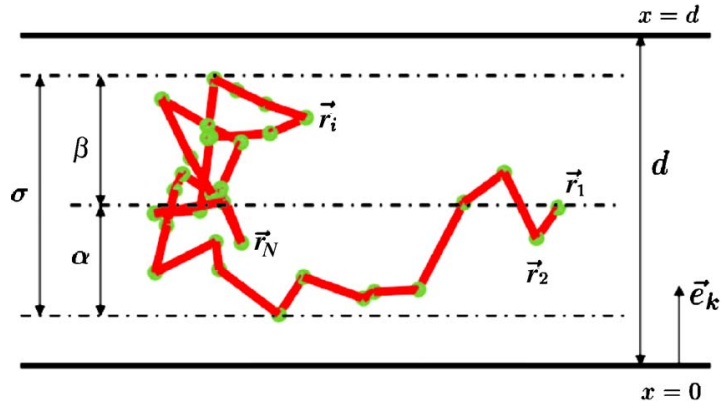

FIG. 1. (Color online) A polymer chain of given configuration $\{\mathbf{R}\}=\{\mathbf{r}\}-\mathbf{r}_{1}$ is confined in a slit of width $d$ and orientation $\mathbf{e}_{k}$. This problem is essentially one dimensional (1D). By projecting the polymer configuration onto $\mathbf{e}_{k}$, we obtain $\sigma$ as the maximum covered distance in $\mathbf{e}_{k}$ direction. In order for this configuration not to be intersected by the slit walls, the first bead can only be located in the region $[\alpha, d-\beta]$ in the $\mathbf{e}_{k}$ direction. The probability that this configuration is not intersected by the slit walls is $(d-\sigma) / d$.

then be taken as the probability that a given molecular internal configuration $\{\mathbf{R}\}$ is not intersected by confining walls given that its first chain segment located anywhere inside the confining geometry $\Omega$.

We see from Eq. (13) that $q(\Omega,\{\mathbf{R}\})$ depends both on the molecular configuration $\{\mathbf{R}\}$ and the confining geometry $\Omega$. However, for certain types of confining geometries such as slits, channels, and boxes, the dependence of $q(\{\mathbf{R}\})$ on the confinement size can be expressed explicitly. This allows the calculation of $K_{0}$ as a function of confinement size from only a single sampling of the internal configurations of a macromolecule under bulk conditions. We therefore name this method the CABS method.

\section{B. The CABS method}

Consider a given polymer configuration defined by internal variables $\{\mathbf{R}\}$ being placed in a slit confining geometry of width $d$, as indicated schematically by Fig. 1 . The slit width $d$ is defined naturally as the distance between two parallel surfaces. Different from the past lattice MC studies, ${ }^{40,41}$ no corrections to $d$ are needed here since we do not discretize the space. A unit vector $\mathbf{e}_{k}$ normal to the slit plane is drawn to define the orientation of the confining slit. We define $\sigma$ as the maximum distance covered by this configuration $\{\mathbf{R}\}$ in the $\mathbf{e}_{k}$ direction, which may be expressed as

$$
\sigma\left(\{\mathbf{R}\}, \mathbf{e}_{k}\right)=\max \left(\{\mathbf{r}\} \cdot \mathbf{e}_{k}\right)-\min \left(\{\mathbf{r}\} \cdot \mathbf{e}_{k}\right) .
$$

$\alpha$ and $\beta$ shown in Fig. 1 are defined as

$$
\begin{aligned}
& \alpha\left(\{\mathbf{R}\}, \mathbf{e}_{k}\right)=\mathbf{r}_{1} \cdot \mathbf{e}_{k}-\min \left(\{\mathbf{r}\} \cdot \mathbf{e}_{k}\right)=-\min \left(\{\mathbf{R}\} \cdot \mathbf{e}_{k}\right), \\
& \beta\left(\{\mathbf{R}\}, \mathbf{e}_{k}\right)=\max \left(\{\mathbf{r}\} \cdot \mathbf{e}_{k}\right)-\mathbf{r}_{1} \cdot \mathbf{e}_{k}=\max \left(\{\mathbf{R}\} \cdot \mathbf{e}_{k}\right) .
\end{aligned}
$$

We see that $\sigma$ is related to $\alpha$ and $\beta$ as

$$
\sigma\left(\{\mathbf{R}\}, \mathbf{e}_{k}\right)=\alpha+\beta=\max \left(\{\mathbf{R}\} \cdot \mathbf{e}_{k}\right)-\min \left(\{\mathbf{R}\} \cdot \mathbf{e}_{k}\right) .
$$

One may notice that in Eq. (17) the absolute position vector of each monomer $\{\mathbf{r}\}$ is used in the definition of $\sigma$. However, since $\sigma$ is a relative measure, it is independent of the choice of $\mathbf{r}_{1}$ as indicated by Eq. (20). We denote by $x$ the location inside the slit of the first chain segment. This polymer con- figuration is not intersected by the slit walls only for $\alpha<x<d-\beta$. We therefore have

$$
\phi\left(\left\{d, \mathbf{e}_{k}\right\},\{\mathbf{R}\}, x\right)=H(x-\alpha) H(d-\beta-x),
$$

where $H(x)$ is the Heaviside function. Substitution of Eq. (21) into Eq. (13) leads to

$$
\begin{aligned}
q\left(\left\{d, \mathbf{e}_{k}\right\},\{\mathbf{R}\}\right) & =\frac{1}{d} \int_{0}^{d} \phi\left(\left\{d, \mathbf{e}_{k}\right\},\{\mathbf{R}\}, x\right) d x \\
& =\left(1-\frac{\sigma}{d}\right) H\left(1-\frac{\sigma}{d}\right) .
\end{aligned}
$$

A function of the form $x H(x)$ is called the Ramp function, $R(x)$, which is equal to $(x+|x|) / 2)$. We therefore rewrite Eq. (22) as

$$
q\left(\left\{d, \mathbf{e}_{k}\right\},\{\mathbf{R}\}\right)=R\left(1-\frac{\sigma}{d}\right) .
$$

The extension of Eq. (23) to channel and box confining geometries is straightforward. We consider a deep channel of rectangular cross section of size $d \times \lambda_{1} d$ and a rectangular box of size $d \times \lambda_{1} d \times \lambda_{2} d$. A deep channel of $d \times \lambda_{1} d$ rectangular cross section can be decomposed into two orthogonal slits of width $d$ and $\lambda_{1} d$, respectively. We take two orthonormal vectors $\mathbf{e}_{k 0}$ and $\mathbf{e}_{k 1}$ to define the orientations of the two slit planes. In this case, $q(\{\mathbf{R}\})$ defined in Eq. (13) reduces to

$$
q\left(\left\{d, \mathbf{e}_{k 0}\right\},\left\{\lambda_{1} d, \mathbf{e}_{k 1}\right\},\{\mathbf{R}\}\right)=R\left(1-\frac{\sigma_{0}}{d}\right) R\left(1-\frac{\sigma_{1}}{\lambda_{1} d}\right),
$$

where $\sigma_{0}$ and $\sigma_{1}$ are obtained according to Eq. (17) for $\mathbf{e}_{k 0}$ and $\mathbf{e}_{k 1}$, respectively. Similarly, for a $d \times \lambda_{1} d \times \lambda_{2} d$ rectangular box confining geometry, $q(\{\mathbf{R}\})$ defined in Eq. (13) reduces to

$$
\begin{aligned}
& q\left(\left\{d, \mathbf{e}_{k 0}\right\},\left\{\lambda_{1} d, \mathbf{e}_{k 1}\right\},\left\{\lambda_{2} d, \mathbf{e}_{k 2}\right\},\{\mathbf{R}\}\right) \\
& \quad=R\left(1-\frac{\sigma_{0}}{d}\right) R\left(1-\frac{\sigma_{1}}{\lambda_{1} d}\right) R\left(1-\frac{\sigma_{2}}{\lambda_{2} d}\right),
\end{aligned}
$$

where $\mathbf{e}_{k 0}, \mathbf{e}_{k 1}$, and $\mathbf{e}_{k 2}$ are three orthonormal vectors defining orientations of the three slits that compose the confining box, and $\sigma_{0}, \sigma_{1}$, and $\sigma_{2}$ are obtained according to Eq. (17).

Substitution of Eqs. (23)-(25) into Eq. (16) leads to the equilibrium partition coefficients for slits, channels, and box confining geometries, respectively. We see from Eqs. (23)-(25) that the information we need from sampling the orientational and conformational freedom of the macromolecule is a series of $\sigma$ data, from which the equilibrium partition coefficient $K_{0}$ can be computed for any confinement size $d$. Besides this superior property in computational speed to previous $\mathrm{MC}$ calculations, CABS also facilitates the identification of possible universal behavior, as presented in the following subsection.

\section{The steric exclusion radius}

We consider the equilibrium partitioning of macromolecules in a slit confining geometry of width $d$ in the dilute solution limit. Substitution of Eq. (23) into Eq. (16) leads to 
TABLE I. The steric exclusion radius $R_{s}$ and root-mean-square radius of gyration $R_{g}$ for some simple rigid objects: A uniform sphere (sphere) and a spherical shell (shell) both of radius $R$; a thin rod (rod) and a rigid dumbbell (dumbbell) both of length $L$; a uniform thin disk (disk) and a rigid ring (ring) both of radius $R$.

\begin{tabular}{ccccccc}
\hline \hline Objects & Sphere & Shell & Rod & Dumbbell & Disk & Ring \\
\hline$R_{s}$ & $R$ & $R$ & $L / 4$ & $L / 4$ & $\pi R / 4$ & $\pi R / 4$ \\
$R_{g}{ }^{a}$ & $\sqrt{3 / 5} R$ & $R$ & $L / \sqrt{12}$ & $L / 2$ & $R / \sqrt{2}$ & $R$ \\
\hline \hline
\end{tabular}

${ }^{\mathrm{a}}$ From Ref. 53.

$$
K_{0}(d)=\left\langle\left(1-\frac{\sigma}{d}\right) H\left(1-\frac{\sigma}{d}\right)\right\rangle .
$$

Equation (26) may be rewritten as an integral over the product of the Ramp function and the probability density function found for $\sigma, f(\sigma)$, and we obtain

$$
\begin{aligned}
K_{0}(d) & =\int_{0}^{\infty}\left(1-\frac{\sigma}{d}\right) H\left(1-\frac{\sigma}{d}\right) f(\sigma) d \sigma \\
& =\int_{0}^{d}\left(1-\frac{\sigma}{d}\right) f(\sigma) d \sigma .
\end{aligned}
$$

The integral in Eq. (27) may be split into

$$
\begin{aligned}
K_{0}(d) & =\int_{0}^{\infty}\left(1-\frac{\sigma}{d}\right) f(\sigma) d \sigma-\int_{d}^{\infty}\left(1-\frac{\sigma}{d}\right) f(\sigma) d \sigma \\
& =1-\langle\sigma\rangle / d+\int_{d}^{\infty}(\sigma / d-1) f(\sigma) d \sigma .
\end{aligned}
$$

The last term in Eq. (28) satisfies $\int_{d}^{\infty}(\sigma / d-1) f(\sigma) d \sigma \geqslant 0$ and vanishes for sufficiently large $d$, in which case we obtain the master equation

$$
K_{0}(d)=1-2 \frac{R_{s}}{d}
$$

where we propose a new molecular size parameter for polymers, the steric exclusion radius $R_{s}$. It is defined as

$$
2 R_{s}=\langle\sigma\rangle=\int_{0}^{\infty} \sigma f(\sigma) d \sigma=\left\langle\max \left(\{\mathbf{r}\} \cdot \mathbf{e}_{k}\right)-\min \left(\{\mathbf{r}\} \cdot \mathbf{e}_{k}\right)\right\rangle
$$

In the following we show how this newly proposed molecular size parameter can be obtained for some simple shaped rigid and flexible macromolecules.

$R_{s}$ for the rigid objects we considered are listed in Table I. Let us, for example, show here how $R_{s}$ can be obtained for a sphere and a thin rod. For a sphere of radius $R, f(\sigma)$ $=\delta(\sigma-2 R)$, where $\delta(x)$ is the Dirac delta function. Therefore, the steric exclusion radius for a sphere is its radius $R_{s}$ $=R$, no matter whether it is a uniform sphere or a spherical shell. The equilibrium partition coefficient with a slit can then be expressed as

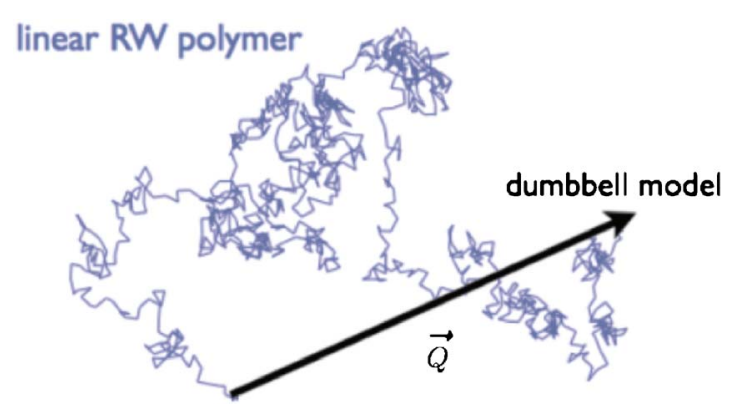

FIG. 2. (Color online) Example of a 3D structure for a flexible linear RW polymer and its coarse-grained model: An elastic dumbbell.

$$
K_{0}(d)=\left\{\begin{array}{l}
1-2 R_{s} / d, \quad \text { for } R_{s} / d \leqslant 1 / 2 \\
0, \quad \text { for } R_{s} / d>1 / 2
\end{array}\right.
$$

We see that the universal partitioning behavior obtained in Eq. (29) is essentially the partitioning behavior of a sphere of radius $R=R_{s}$. For a thin rod of length $L$, it can be shown by assuming an isotropic orientation of the rod that $f(\sigma)$ is a continuous uniform distribution of the form

$$
f(\sigma)=\left\{\begin{array}{l}
1 / L, \quad \text { for } 0 \leqslant \sigma \leqslant L \\
0, \quad \text { for } \sigma>L
\end{array}\right.
$$

Substitution of Eq. (32) into Eq. (30) leads to the steric exclusion radius for a thin rod of length $L$. We obtain $R_{s}$ $=L / 4$. The equilibrium partition coefficient $K_{0}$ for a thin rod of length $L$ with a confining slit of width $d$ can be obtained by inserting Eq. (32) into Eq. (27), which leads to

$$
K_{0}(d)= \begin{cases}1-2 R_{s} / d, & \text { for } R_{s} / d \leqslant 1 / 4 \\ \left(R_{s} / d\right)^{-1} / 8, & \text { for } R_{s} / d>1 / 4\end{cases}
$$

We see that $K_{0}$ for a rod follows the master equation (29) for $R_{s} / d \leqslant 1 / 4$.

We compare in Table I the steric exclusion radius $R_{s}$ and root-mean-square radius of gyration $R_{g}$ for some simple rigid objects of sphere, rod, and disk shapes. In particular, comparisons are made between (i) a uniform sphere and a spherical shell, (ii) a uniform rod and a rigid dumbbell, and (iii) a uniform disk and a rigid ring. It is clear that for each pair $R_{g}$ are different from each other due to the fact that $R_{g}$ is defined as the root-mean-square distance of the collection of mass points from their common center of mass. However, $R_{s}$ are the same for each pair, since it is only related to the mass points that are located on the surface of each molecular configuration according to Eq. (30). As first pointed out by Giddings et al., ${ }^{14}$ steric exclusion may essentially be a surface effect. We may thus expect that $R_{s}$ is a more relevant molecular length scale than $R_{g}$ for characterization of spatial confinement effects on macromolecules.

Flexible polymers have both orientational and conformational degrees of freedom. The simplest model of a flexible polymer would be an elastic dumbbell. It includes two point beads connected by a vector $\mathbf{Q}$, as shown in Fig. 2, and the connection vector $\mathbf{Q}$ follows a Gaussian distribution ${ }^{42}$ 


$$
p(\mathbf{Q})=\left(\frac{3}{2 \pi\left(\mathbf{Q}^{2}\right)}\right)^{3 / 2} \exp \left(-\frac{3 \mathbf{Q}^{2}}{2\left\langle\mathbf{Q}^{2}\right\rangle}\right) .
$$

The distribution function of $\sigma$ defined in Eq. (17) is found to be

$$
f(\sigma)=\left(\frac{6}{\pi\left\langle\mathbf{Q}^{2}\right\rangle}\right)^{1 / 2} \exp \left(-\frac{3 \sigma^{2}}{2\left\langle\mathbf{Q}^{2}\right\rangle}\right) .
$$

Substitution of Eq. (35) into Eq. (30) leads to the steric exclusion radius for an elastic dumbbell of mean-square endto-end distance $\left\langle\mathbf{Q}^{2}\right\rangle$,

$$
R_{s}=\frac{1}{\sqrt{6 \pi}}\left\langle\mathbf{Q}^{2}\right\rangle^{1 / 2},
$$

and the equilibrium partition coefficient for an elastic dumbbell with a slit of width $d$ is found by substituting Eq. (35) into Eq. (27),

$$
K_{0}(d)=\operatorname{erf}\left(\frac{1}{2 \sqrt{\pi}} \frac{d}{R_{s}}\right)-\frac{2 R_{s}}{d}\left[1-\exp \left(-\frac{d^{2}}{4 \pi R_{s}^{2}}\right)\right],
$$

where $\operatorname{erf}(x)=(2 / \sqrt{\pi}) \int_{0}^{x} \exp \left(-t^{2}\right) d t$ is the error function. If we use the large $x$ expansion of the error function, we get

$$
\begin{aligned}
K_{0}(d)= & 1-2 \frac{R_{s}}{d}+\exp \left(-\frac{d^{2}}{4 \pi R_{s}^{2}}\right) \\
& \times \sum_{n=1}^{\infty}(-1)^{n-1} \pi^{n}(2 n-1) ! !\left(\frac{2 R_{s}}{d}\right)^{2 n+1},
\end{aligned}
$$

where $(2 n-1) ! !=1 \cdot 3 \cdot 5 \cdots(2 n-1)$. We see from Eq. (38) that the master equation given in Eq. (29) is recovered for $R_{S} / d \rightarrow 0$.

For a linear random walk polymer of $N$ point beads and step length $b$, a direct analytical derivation of $f(\sigma)$ may be difficult. Therefore, we have developed an alternative procedure to obtain $f(\sigma)$ in the long chain limit. From Eq. (26), we obtain

$$
\frac{\partial K_{0}}{\partial d}=\left\langle\frac{\sigma}{d^{2}} H\left(1-\frac{\sigma}{d}\right)\right\rangle,
$$

and by combining Eqs. (26) and (39), we obtain

$$
K_{0}+d \frac{\partial K_{0}}{\partial d}=\left\langle H\left(1-\frac{\sigma}{d}\right)\right\rangle=\int_{0}^{d} f(\sigma) d \sigma \equiv F(d),
$$

where $F(d)$ is the probability that variable $\sigma$ lies in the interval $[0, d]$. Note that an explicit expression of $\left(K_{0}\right.$ $\left.+d \partial K_{0} / \partial d\right)$ can be obtained from the expression of $K_{0}(d)$ derived by Casassa, ${ }^{1}$ who showed that the equilibrium partition coefficient follows

$$
K_{0}(d)=\frac{8}{\pi^{2}} \sum_{m=0}^{\infty} \frac{\exp \left[-(2 m+1)^{2} \pi^{2} R_{g}^{2} d^{-2}\right]}{(2 m+1)^{2}},
$$

for a linear flexible RW polymer of radius of gyration $R_{g}$ with a confining slit of width $d$. Substitution of Eq. (41) into Eq. (40) leads to

$$
\begin{aligned}
F(d)= & \frac{8}{\pi^{2}} \sum_{m=0}^{\infty}\left[(2 m+1)^{-2}+2 \pi^{2} R_{g}^{2} d^{-2}\right] \\
& \times \exp \left[-(2 m+1)^{2} \pi^{2} R_{g}^{2} d^{-2}\right] .
\end{aligned}
$$

The function $F$ is called the cumulative distribution function, and the probability density function $f(\sigma)$ is obtained by taking the derivative of $F(\sigma)$ with respect to $\sigma$. We get

$$
\begin{aligned}
f(\sigma)=\frac{d F(\sigma)}{d \sigma}= & \sum_{m=0}^{\infty}\left[32 \pi^{2}(2 m+1)^{2} R_{g}^{4} \sigma^{-5}-16 R_{g}^{2} \sigma^{-3}\right] \\
& \times \exp \left[-(2 m+1)^{2} \pi^{2} R_{g}^{2} \sigma^{-2}\right] .
\end{aligned}
$$

Note that for a linear random walk polymer of $N$ point beads and step length $b, \sigma$ cannot be larger than $L=(N-1) b$, which is the contour length of the chain. In the long chain limit, we obtain $R_{s}$ as

$$
2 R_{s}=\lim _{L \rightarrow \infty} \int_{0}^{L} \sigma f(\sigma) d \sigma .
$$

Substitution of Eq. (43) into Eq. (44) leads to

$$
R_{s}=\frac{2}{\sqrt{\pi}} R_{g}=\frac{2}{\sqrt{6 \pi}}\left\langle\mathbf{Q}^{2}\right\rangle^{1 / 2},
$$

where $\left\langle\mathbf{Q}^{2}\right\rangle=6 R_{g}^{2}$ is the mean-square end-to-end distance for a linear RW polymer in the long chain limit.

It is interesting to notice from Eqs. (36) and (45) that the steric exclusion radius of a linear long chain RW polymer is two times the $R_{s}$ value for an elastic dumbbell with the same $\left\langle\mathbf{Q}^{2}\right\rangle$. Moreover, the relation between $R_{s}$ and $R_{g}$ for a linear RW polymer in the long chain limit, which reminds us of the classical Asakura-Oosawa (AO) model, ${ }^{43,44}$ where ideal polymer chains are modeled as interpenetrable spheres of radii $R_{\mathrm{AO}}$. It was pointed out by Bolhuis et al. ${ }^{45}$ and Louis et al. ${ }^{46}$ that one should take $R_{\mathrm{AO}}=(2 / \sqrt{\pi}) R_{g}$ instead of $R_{\mathrm{AO}}$ $=R_{g}$ by considering the concentration depletion effect for a dilute polymer solution confined in a slit.

We see that analytical derivations of $f(\sigma)$ and $R_{S}$ even for a linear RW polymer can be difficult. However, with the rapid advances in computing facilities and molecular simulation techniques, a numerical determination of these quantities is rather simple by sampling the configuration space of a macromolecule using e.g. Monte Carlo simulations.

\section{SIMULATION}

A numerical implementation of our CABS method for the computation of the equilibrium partition coefficient $K_{0}(d)$ is straightforward. It only requires a simulation technique that samples the configuration space of the studied macromolecule in bulk. For each molecular configuration sampled, one can obtain $\left\{\sigma_{0}, \sigma_{1}, \sigma_{2}\right\}$ from the definition of $\sigma$ given in Eq. (17) by sampling three orthonormal vectors $\left\{\mathbf{e}_{k 0}, \mathbf{e}_{k 1}, \mathbf{e}_{k 2}\right\}$ of isotropically random orientation. This process generates a sequence of $\sigma$ data which are then used for calculating the equilibrium partition coefficients for slits, channels, and box confining geometries as a function of confinement size $d$ based on Eqs. (23)-(25). A histogram analysis of the $\sigma$ data gives $f(\sigma)$, and a simple average of $\sigma$ gives twice of the 
steric exclusion radius $R_{s}$. Additionally, a number of conventional molecular size parameters can be obtained from sampling bulk configurations. For comparison with the steric exclusion radius, we calculated the mean-square radius of gyration and Zimm-Kirkwood hydrodynamic radius ${ }^{16}$

$$
\begin{aligned}
& R_{g}^{2}=\frac{1}{2} \frac{1}{N^{2}} \sum_{m=1}^{N} \sum_{n=1}^{N}\left\langle\left(\mathbf{r}_{n}-\mathbf{r}_{m}\right)^{2}\right\rangle, \\
& R_{h}^{-1}=\frac{1}{N^{2}} \sum_{m \neq n}\left\langle\left|\mathbf{r}_{n}-\mathbf{r}_{m}\right|^{-1}\right\rangle,
\end{aligned}
$$

where $\mathbf{r}_{n}$ is the position vector of the $n$th bead in the molecule that consists of $N$ beads. Note that although the derivation of Eq. (47) involves assumptions about the hydrodynamic interactions between beads for a bead-spring chain, ${ }^{3}$ one may, however, regard $R_{h}$ as a molecular size parameter that is defined differently from $R_{g}$.

We applied the CABS method to study the equilibrium partitioning behavior of linear RW and SAW polymers. Similar to the chain generation method used by Davidson et al. ${ }^{17}$ and Tobita et al., ${ }^{47}$ a linear RW polymer of $N$ point beads was generated by performing random walk for $N-1$ steps with fixed step length. This method was also used to generate conformations for a nonlinear RW polymer by drawing each primary chain as the trajectory of the random walk in the three-dimensional (3D) space. A linear SAW polymer was sampled on a cubic lattice by the pivot algorithm using a code implemented by Kennedy, ${ }^{48}$ which requires a time $\mathcal{O}\left(N^{q}\right)$ with $q<1$ per accepted pivot. It is worth mentioning that there exist various models and levels of descriptions for a polymer chain, and our CABS method for obtaining the equilibrium partition coefficients is easily adaptable to different models and types of the macromolecules as long as purely steric exclusion effects are considered.

The equilibrium partition coefficient is obtained from CABS as an average over the rotational and conformation degrees of freedom of a macromolecule. The accuracy of the results thus depends on the total number of conformations sampled and the number of orientations sampled per chain conformation. In principle, the more degrees of freedom of the macromolecule, the more conformations should be sampled to ensure accuracy of the results. The total number of chain conformations sampled ranged from $10^{4}$ to $10^{6}$. For each conformation, 10 sets of orientations were sampled, and each set had three orthonormal vectors. We repeated each calculation ten times to estimate the statistical error. ${ }^{49}$ The statistical errors of our simulation results were typically of the order of the line width in the plots and are therefore not shown.

\section{RESULTS AND DISCUSSIONS}

\section{A. Bulk structure properties}

It is well known that the average size of an isolated uncharged flexible polymer chain in solution is asymptotically proportional to $N^{\nu}$, where $N$ is the total number of monomers and $\nu$ is the Flory exponent with $\nu=1 / 2$ for $\Theta$-solvent condition and $\nu \approx 0.5877$ for good solvent condi-

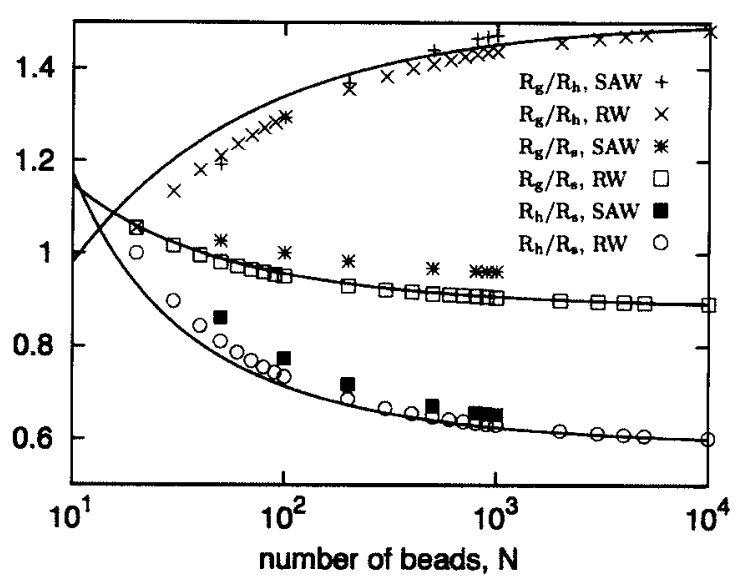

FIG. 3. Characteristic ratios $R_{g} / R_{h}, R_{g} / R_{s}$, and $R_{h} / R_{s}$ obtained from linear RW and SAW simulations are plotted as a function of the total number of beads, $N$. Solid lines are predictions based on Eqs. (48)-(50).

tion. This law holds for any measure of the chain size, but is valid only in the asymptotic limit $N \rightarrow \infty$. For finite $N$, deviations occur, and corrections have been considered for both $R_{g}$ and $R_{h}$. For a linear RW polymer of $N$ mass points with step length $b$, Dünweg et al. ${ }^{50}$ showed that

$$
\begin{aligned}
& \left\langle R_{g}^{2}\right\rangle=\frac{1}{6} N b^{2}\left(1-N^{-2}\right), \\
& \left\langle R_{h}^{-1}\right\rangle=A b^{-1} N^{-1 / 2}\left[1-B N^{-1 / 2}-\mathcal{O}\left(N^{-3 / 2}\right)\right],
\end{aligned}
$$

where $A=(8 / 3) \sqrt{6 / \pi}$ and $B \approx 1.095266$. We considered corrections to the steric exclusion radius $R_{S}$ when $N$ is finite and found from our linear RW simulations that an expression of the form

$$
R_{S} / b \approx 0.4607 N^{1 / 2}\left(1-0.7329 N^{-1 / 2}\right)
$$

gave a reasonably good fit to the simulation results, and Eq. (45) was also recovered in the long chain limit.

In Fig. 3, three characteristic structure ratios $R_{g} / R_{h}$, $R_{g} / R_{s}$, and $R_{h} / R_{s}$ are plotted as a function of $N$ for both linear RW and SAW chains. Also plotted are predictions based on Eqs. (48)-(50). Clearly, all predictions agree well with our linear RW simulations for large $N$. For small $N$, simulation results for $R_{h}$ are larger than predicted by Eq. (49), which accounts for the difference between simulations results and theoretical prediction for both $R_{g} / R_{h}$ and $R_{h} / R_{s}$ when $N$ is less than about $10^{3}$ in Fig. 3. For both linear RW and SAW chains, $R_{g} / R_{h}$ increases as $N$ increases, while the opposite trend is observed for both $R_{g} / R_{s}$ and $R_{h} / R_{s}$. Noteworthy, all three ratios are smaller for a linear RW chain than for a linear SAW chain of the same $N$. However, the ratio $R_{h} / R_{s}$ seems to have a smaller difference between a linear SAW chain and a linear RW chain than the ratio $R_{g} / R_{s}$. The results shown in Fig. 3 are important in understanding the difference among $R_{g}, R_{h}$, and $R_{s}$ when they are used as the representative molecular size parameter in the description of the equilibrium partitioning behavior as presented in the following subsections.

We also calculated the distribution function of $\sigma$ from our MC simulations by a simple histogram analysis. As shown in Fig. 4, distribution functions $f\left(\sigma / R_{s}\right)$ calculated for a rigid rod, an elastic dumbbell, and a linear RW polymer of 


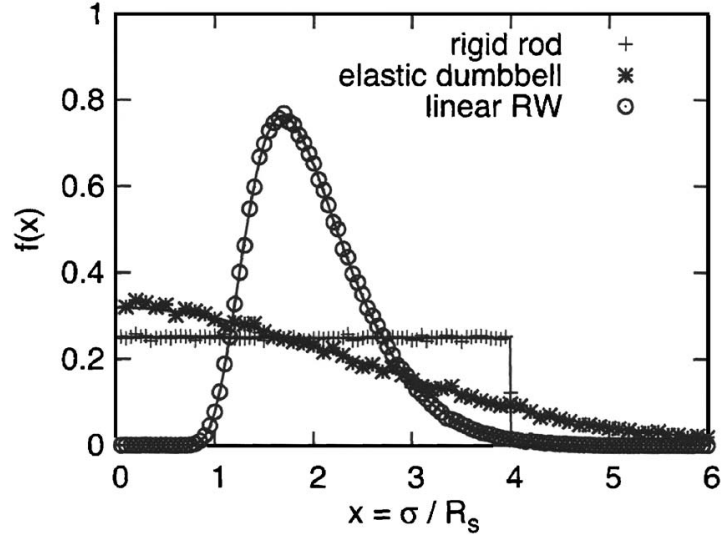

FIG. 4. Distribution functions of $\sigma / R_{s}$ are shown for a rigid rod, an elastic dumbbell, and a linear RW polymer of $10^{4}$ beads. Points are our simulations results, and solid lines are predictions based on Eqs. (32), (35), and (43).

$10^{4}$ point beads from our MC simulations agree well with theoretical predictions based on Eqs. (32), (35), and (43). It is interesting to notice the large difference in the distribution function of $\sigma / R_{s}$ between an elastic dumbbell and a linear RW chain. We also see that the condition of "sufficiently large $d$ " for Eq. (29) to be valid is satisfied when $d>4 R_{s}$ for a rigid rod, while it is not the case for an elastic dumbbell. In contrast, it seems to be sufficient for a linear RW polymer.

\section{B. Comparison with prior studies}

In this subsection, we validate our CABS calculations of $K_{0}$ by comparing them to previous determinations. Results are presented in the traditional way where $K_{0}$ is plotted as a function of the ratio of the radius of gyration to the confinement size, $R_{g} / d$. The effects of confining geometries, chain flexibility, and solvent quality on the equilibrium partitioning behavior are also considered.

Quantitative studies of the equilibrium partition coefficients of macromolecules were initiated by Casassa, ${ }^{1}$ who derived expressions for $K_{0}$ for a long chain linear RW polymer with cavities of spherical, cylindrical, and slit shapes. Cifra and Teraoka ${ }^{29}$ pointed out later that the equilibrium partition coefficients for ideal polymers with slit, square channel and cubic box confining geometries were closely related due to the fact that confinement is independent in each confinement direction, and for the same $R_{g} / d$, one has

$$
K_{0, \text { slit }}=K_{0, \text { square channel }}^{1 / 2}=K_{0, \text { cubic box }}^{1 / 3} \cdot
$$

Equation (51) can be derived from Eqs. (23)-(25) by assuming that $\sigma_{0}, \sigma_{1}$, and $\sigma_{2}$ are not correlated. The equation certainly also holds for a spherical molecule where $\sigma_{0}=\sigma_{1}=\sigma_{2}$, but does not hold, e.g., for a rod molecule where $\sigma_{0}, \sigma_{1}$, and $\sigma_{2}$ are strongly correlated but not identical.

In Fig. 5, the equilibrium partition coefficients are shown for a long chain linear RW polymer with a slit confining geometry of width $d$, a square channel of cross section $d \times d$, a cylindrical pore of diameter $d$, a cubic box of size $d \times d \times d$, and a spherical cavity of diameter $d$. For the same confinement size $d$, it is clear that among these confining geometries $K_{0}$ are ordered as: slit $>$ square channel $>$ cylindrical pore $>$ cubic box $>$ spherical cavity. For slit,

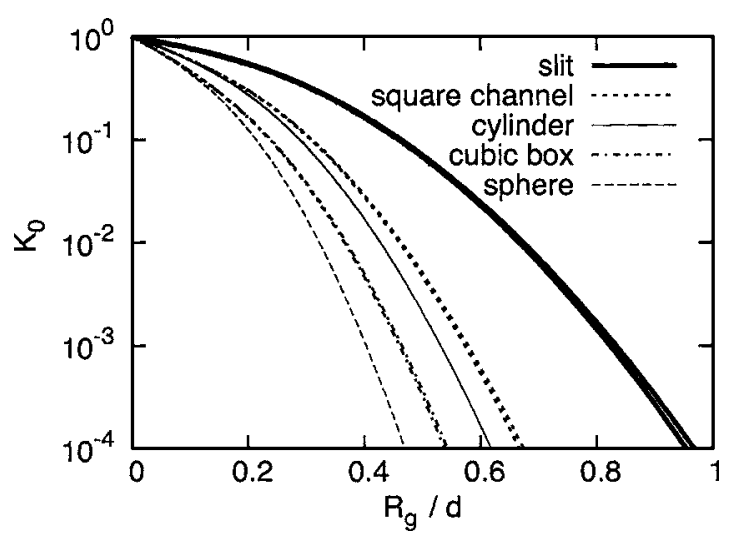

FIG. 5. Equilibrium partition coefficients $K_{0}$ as a function of $R_{g} / d$ are shown for a long chain linear RW polymer with (from top to bottom) a slit of width $d$, a long square channel of $d \times d$ cross section, a long cylinder of diameter $d$, a cubic box of size $d \times d \times d$, and a sphere of diameter $d$. Both our simulation results and analytical results given in Eqs. (41) and (51) are shown for slit, square channel, and cubic box confining geometries, and they nearly coincide with one another even for $K_{0}$ as small as $10^{-4}$. For cylinder and sphere confining geometries, only Casassas results are shown (Ref. 1).

square channel, and cubic box confining geometries, our simulation results for a linear RW polymer of $10^{4}$ point beads agree well with predictions based on Eqs. (41) and (51), so that they are not distinguishable in the figure.

Casassa's approach ${ }^{1,15,16}$ is based on solving the diffusion equation of a RW chain, and therefore the results are valid only for the limiting case where $N \rightarrow \infty$ for a real chain of finite segment length. As shown in Fig. $6, K_{0}$ for a linear RW chain of finite number of steps can be substantially higher than predicted from Eq. (41), which is in agreement with the MC simulation results by Davidson et al. ${ }^{17}$ Simulation results for $N=2$ agree with the analytical solution for a rigid dumbbell derived by Giddings et al. ${ }^{14}$ Note that the partitioning behavior of a rigid dumbbell of length $L$ is essentially the same as that of a uniform rigid rod of the same length except that for a rigid dumbbell $R_{g}=L / 2$, while for a uniform rigid $\operatorname{rod} R_{g}=L / \sqrt{12}$, as listed in Table I.

The finite $N$ effect reflects the effect of chain flexibility

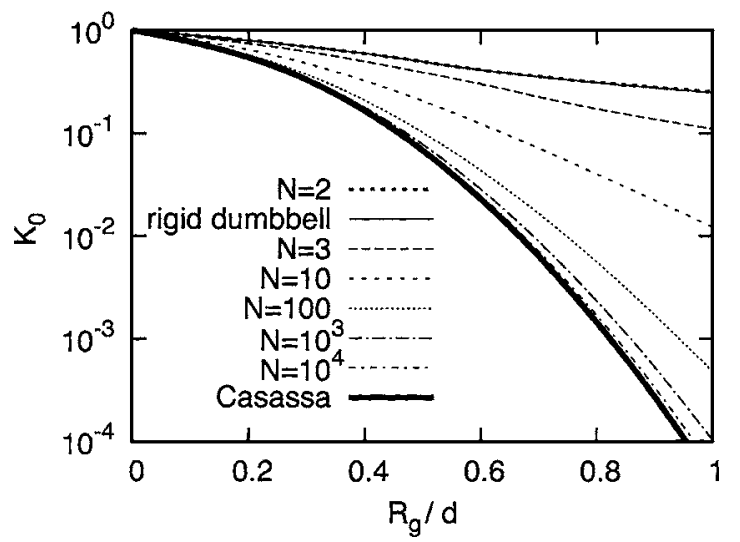

FIG. 6. Equilibrium partition coefficients $K_{0}$ as a function of $R_{g} / d$ are shown for linear RW polymers of $N$ beads with a slit confining geometry of width $d$. From top to bottom: $N=2,3,10,10^{2}, 10^{3}, 10^{4}$, and $N=\infty$. The analytical solution for a rigid dumbbell (Ref. 14) is also plotted, which coincides with the simulation results of $N=2$. Casassa's result (Ref. 1) given by Eq. (41) corresponds to $N=\infty$. 


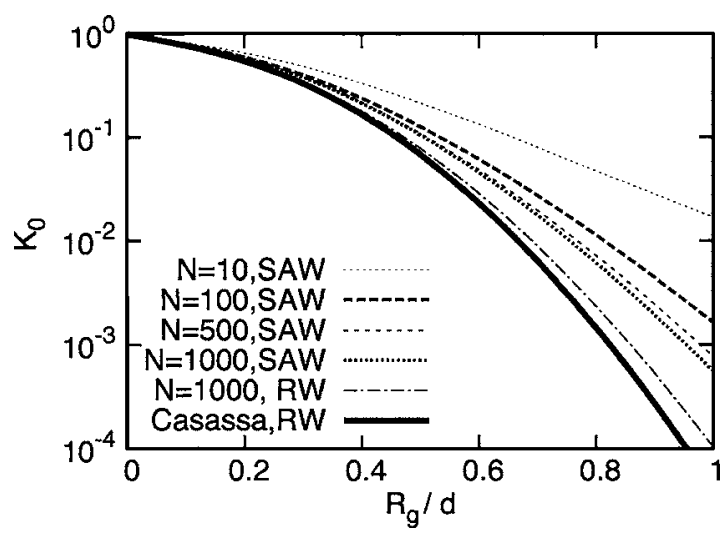

FIG. 7. Equilibrium partition coefficients $K_{0}$ as a function of $R_{g} / d$ are shown for linear SAW polymers of $N$ beads with a slit confining geometry of width $d$. From top to bottom: $N=10,100,500,10^{3}$, simulation results of a linear RW chain of $N=10^{3}$, and Casassa's result (Ref. 1) given by Eq. (41) corresponding to a linear RW chain of $N=\infty$.

on the equilibrium partitioning behavior of polymers. As one may anticipate, this effect is also observed in the equilibrium partitioning of linear SAW chains. As shown in Fig. 7, for the same $R_{g} / d$ value, $K_{0}$ decreases as $N$ increases. The difference in the partitioning behavior of linear RW and SAW chains are also demonstrated in Fig. 7. For polymers of the same $R_{g} / d, K_{0}$ of a linear SAW chain is higher than that of a linear RW chain, which is in agreement with the simulation results by Cifra et $a{ }^{22}$ and Bleha et $a .^{23}$ These may be understood from Fig. 3 since a linear SAW polymer would have a smaller $R_{S}$ than a linear RW polymer of the same $N$ and $R_{g}$ and thus would have a larger $K_{0}$ for the same confinement size $d$ according to Eq. (29). The same argument holds for the finite $N$ effect for two linear RW polymers of the same $R_{g}$. The polymer of a smaller $N$ would have a smaller $R_{S}$ resulting in a larger $K_{0}$ for the same confinement size $d$.

The excellent agreement between available theoretical results and our calculations validates our CABS method. In addition, we may obtain a "continuous" curve of $K_{0}$ as a function of $d$ from just one set of simulations since they are independent of $d$. This is in contrast to previous MC calculations of $K_{0}$ which only were done for a discrete set of $d$ values, since for each $d$ value a new set of simulations was required.

\section{A universal partitioning behavior}

A persistent interest in theoretical determinations of $K_{0}$, among many others, is to understand the separation principle of SEC, i.e., upon what general size basis, if there is one, does SEC separate macromolecules? Based on the equilibrium partitioning theory, the elution volume of polymer molecules from a SEC column is a function of the equilibrium partition coefficient, ${ }^{14,15,36}$ and the challenge is therefore to find a single molecular size parameter, if it exists, that may be used to determine $K_{0}$ for any chain architecture in a given confinement. Indeed, despite the many successes of the equilibrium partitioning theory in understanding the elution behavior of polymers in SEC, this problem has remained unsolved. Clearly, the root-mean-square radius of gyration $R_{g}$

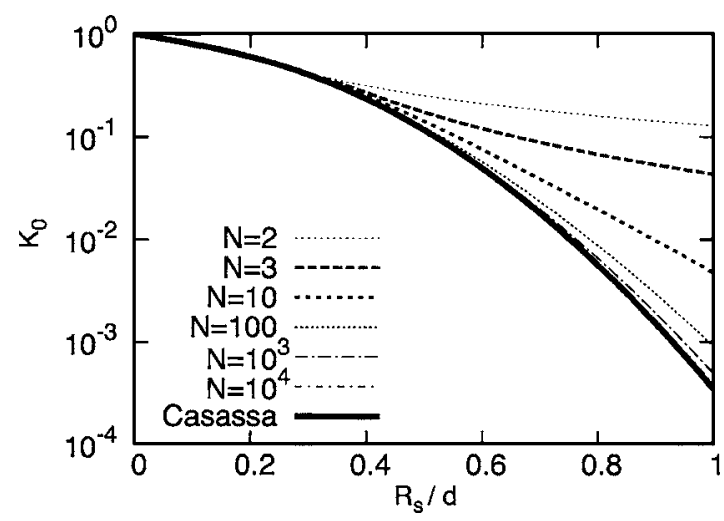

FIG. 8. Equilibrium partition coefficients $K_{0}$ as a function of $R_{s} / d$ are shown for linear RW polymers of $N$ beads with a slit confining geometry of width $d$. From top to bottom: $N=2,3,10,10^{2}, 10^{3}, 10^{4}$, and $N=\infty$. Casassa's result (Ref. 1) given by Eq. (41) corresponds to $N=\infty$, which nearly coincides with the simulation results for $N=10^{4}$.

alone could not be the determining size parameter, as shown in Figs. 6 and 7. For two polymers of the same $R_{g} / d$ value, a linear SAW polymer would have a larger $K_{0}$ than a linear RW polymer; a linear RW polymer of finite number of segments would have a larger $K_{0}$ than that of infinite number of segments. In addition, a linear RW polymer would have a larger $K_{0}$ than a nonlinear RW polymer as first noticed by Casassa and Tagami. ${ }^{15}$

The reason we think that the use of $R_{g}$ does not lead to universal partitioning behavior lies in the fact that $R_{g}$, as defined in Eq. (46), depends on all the mass points of the molecule, while steric exclusion may only be a surface effect. ${ }^{14}$ This can be illustrated by considering two simple examples. Naturally, one would anticipate that a uniform sphere and a spherical shell of the same radius $R$ with a confining slit of width $d$ have the same $K_{0}$. However, they will not have the same $K_{0}$ if they have the same radius of gyration $R_{g}$, since for a uniform sphere $R_{g}=\sqrt{3 / 5} R$ while for a spherical shell $R_{g}=R$. The same argument holds, e.g., for the partitioning behavior of a uniform rigid rod and an elastic dumbbell of the same length. In the following, we show that a better universal relationship is indeed obtained with the new molecular size parameter $R_{s}$.

First of all, we would like to examine the universality in the equilibrium partitioning behavior for the same data, as shown in Figs. 6 and 7. We see in Figs. 8 and 9 that, when $K_{0}$ is shown as a function of $R_{s} / d$ instead, a universal regime appears for approximately $R_{s} / d<0.25$. As long as $R_{s} / d$ stays in the universal regime, the finite $N$ effect and the change from RW to SAW conformation statistics only affect the specific $R_{s}$ value, but do not alter the equilibrium partitioning behavior, i.e., $K_{0}$ follows Eq. (29) for a slit confining geometry of width $d$. Since Eq. (29) is obtained under the assumption that the confinement size $d$ is sufficiently large, deviations from the master curve are expected as $R_{s} / d$ increases. It is also noticed that among all the results shown in Fig. 8 a rigid dumbbell $(N=2)$ deviates first from the master curve, and we know from Eq. (33) that deviation appears at $R_{s} / d$ $=1 / 4$ for a rigid dumbbell.

Previously, $R_{g}, R_{g} g^{-1 / 3}$, and $R_{h}$ were proposed as the representative molecular size parameter in the description of 


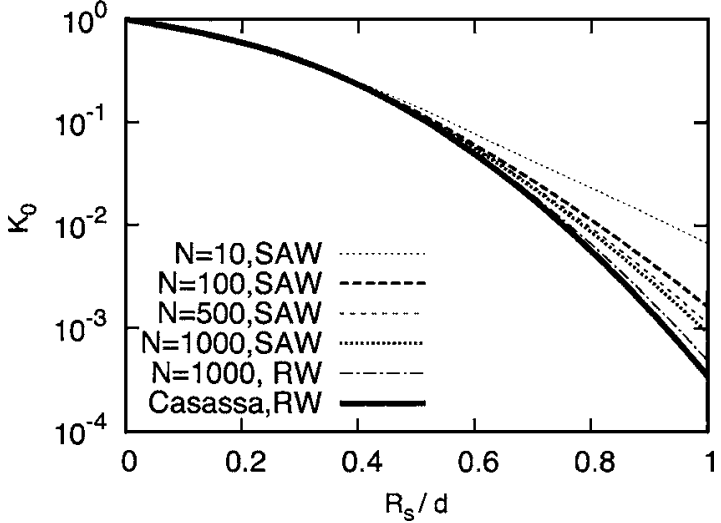

FIG. 9. Equilibrium partition coefficients $K_{0}$ as a function of $R_{s} / d$ are shown for linear SAW polymers of $N$ beads with a slit confining geometry of width $d$. From top to bottom: $N=10,100,500,10^{3}$, simulation results of a linear RW chain of $N=10^{3}$, and Casassa's result (Ref. 1) given by Eq. (41) corresponding to a linear RW chain of $N=\infty$.

the equilibrium partitioning of macromolecules. ${ }^{15,16}$ In Fig. $10, K_{0}$ is shown as a function of $R_{g} / d, R_{g} g^{-1 / 3} / d, R_{h} / d$, and $R_{S} / d$, respectively, for a linear SAW polymer of $N=10^{3}$, a linear RW polymer of $N=10^{3}$, a symmetric three-arm RW star polymer of arm length $N=10^{3}$, and a symmetric fourarm RW star polymer of arm length $N=10^{3}$ with a slit confining geometry of width $d$. Different from Figs. 6-9, a linear scale of $K_{0}$ is used in Fig. 10, since it is understood from the equilibrium partitioning theory for SEC that the elution volume depends on $K_{0}$ linearly. ${ }^{15}$ It is clear that, compared to the use of $R_{g}$, the use of $R_{g} g^{-1 / 3}$ or $R_{h}$ is capable of providing better universality in the partitioning behavior. However, the best universality is obtained by the use of our new molecular size parameter, $R_{s}$. We see in Fig. 10(d) that when $K_{0}$ is shown as a function of $R_{s} / d$, results for the four flexible polymers (linear SAW, linear RW, three-arm RW star, and four-arm RW star) are nearly indistinguishable.

We expect from Fig. 4 that an elastic dumbbell may deviate from the master curve at a smaller $R_{s} / d$ value than $1 / 4$. This is verified in Fig. 10(d), where additionally the equilibrium partition coefficients $K_{0}$ are shown as a function of $R_{S} / d$ for an elastic dumbbell, a rigid rod, and a sphere with a slit confining geometry of width $d$. It is interesting to notice from Fig. 10(d) that the equilibrium partitioning behavior of an elastic dumbbell deviates largely from that of a long chain linear RW polymer. We already pointed out in Eqs. (36) and (45) that for two molecules of the same meansquare end-to-end distance $\left\langle\mathbf{Q}^{2}\right\rangle$, an elastic dumbbell has only half of the steric exclusion radius of a long chain linear RW polymer. We therefore conclude that an elastic dumbbell model over predicts $K_{0}$ of a linear RW polymer of the same $\left\langle\mathbf{Q}^{2}\right\rangle$ especially in the high confinement regime.

Consistent with Eq. (29), we see a unique curve as $R_{s} / d \rightarrow 0$ in Fig. 10(d). As $R_{s} / d$ increases, deviations from Eq. (29) appear. This may lead to a natural clarification of the so-called weak and high confinement regimes. In the weak confinement regime, $K_{0}$ depends only on $R_{s} / d$ regardless of the chain architecture and conformation statistics, while in the high confinement regime, there is no universal partitioning behavior observed when $K_{0}$ is shown as a function of $R_{s} / d$. We know from Eqs. (31) and (33) that a sphere follows the universal curve for the entire $K_{0}$ window from 0 to 1 , while a rigid rod deviates from the universal behavior for $K_{0}<1 / 2$ and follows $\left(R_{s} / d\right)^{-1 / 8}$ instead. As shown in Fig. 10(d), the equilibrium partitioning curves $K_{0}\left(R_{s} / d\right)$ for long chain polymers in the high confinement regime lie between that of a rod and a sphere, and may be described by the scaling theory $K_{0} \sim-\left(R_{s} / d\right)^{1 / \nu},{ }^{18,20}$ where $\nu$ is the Flory exponent.
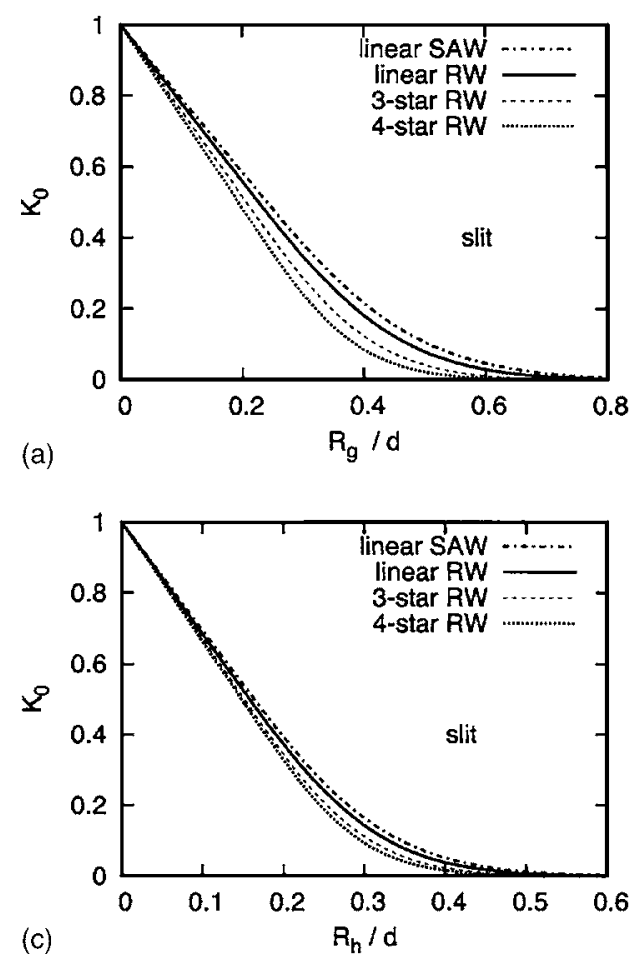
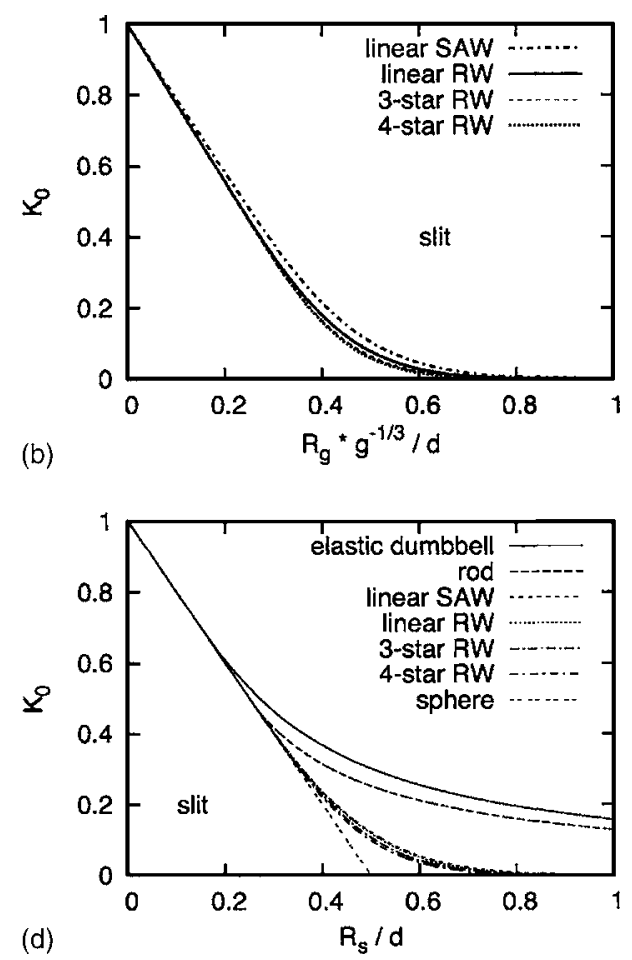

FIG. 10. Equilibrium partition coefficients $K_{0}$ are shown (a) as a function of $R_{g} / d$, (b) as a function of $R_{g} g^{-1 / 3} / d$, (c) as a function of $R_{h} / d$, and (d) as a function of $R_{s} / d$ for (from top to bottom) a linear SAW polymer of $N$ $=10^{3}$, a linear RW polymer of $N=10^{3}$, a symmetric three-arm RW star polymer of arm length $N=10^{3}$ with $g$ $=0.778$, and a symmetric four-arm RW star polymer of arm length $N=10^{3}$ with $g=0.625$ with a slit confining geometry of width $d$. In subfigure (d), $K_{0}$ are also shown for an elastic dumbbell, a rigid rod, and a sphere. 


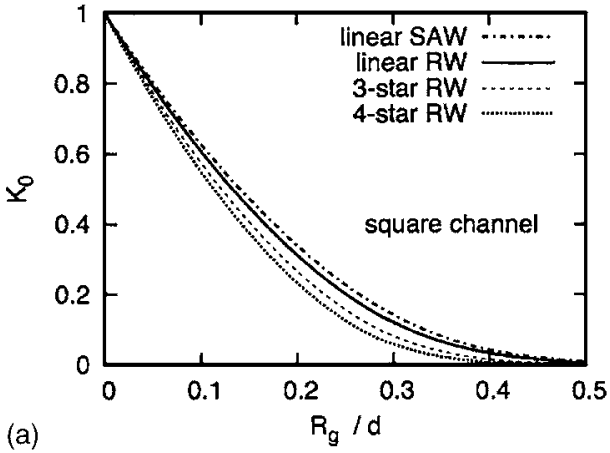

(a)

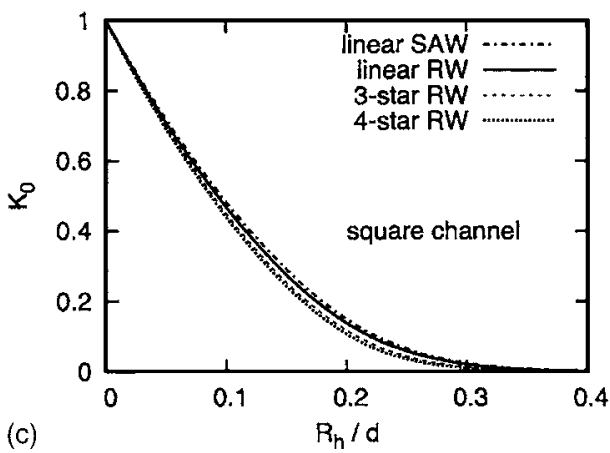

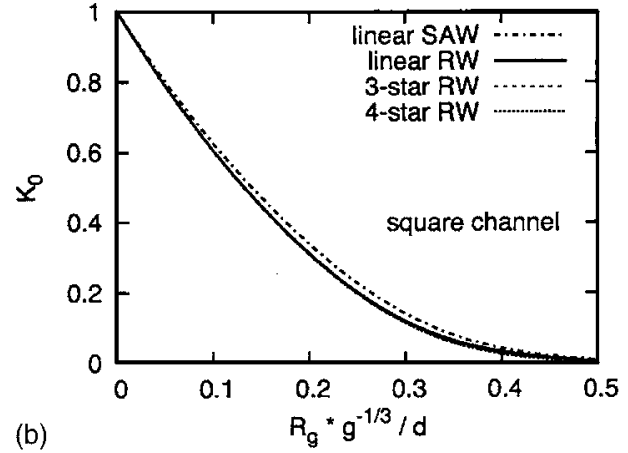

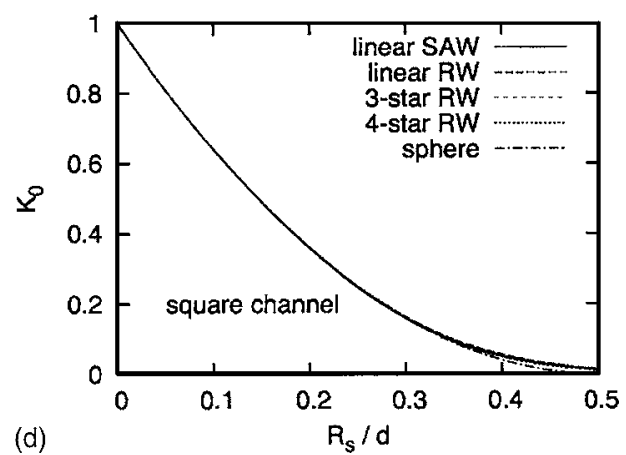

FIG. 11. Equilibrium partition coefficients $K_{0}$ are shown (a) as a function of $R_{g} / d$, (b) as a function of $R_{g} g^{-1 / 3} / d$, (c) as a function of $R_{h} / d$, and (d) as a function of $R_{S} / d$ for (from top to bottom) a linear SAW polymer of $N$ $=10^{3}$, a linear RW polymer of $N=10^{3}$, a symmetric three-arm RW star polymer of arm length $N=10^{3}$, and a symmetric four-arm RW star polymer of arm length $N=10^{3}$ with a square channel confining geometry of $d \times d$ cross section. In subfigure (d), $K_{0}$ for a sphere is also shown.
Note that the equilibrium partition coefficients shown in Fig. 10 are for a slit confining geometry. Naturally, one may expect that the confinement effect in an SEC column is two dimensional (2D) and the confining geometry may be effectively described by a square channel. Therefore, it is worthwhile to investigate the universality in the partitioning behavior with $2 \mathrm{D}$ and $3 \mathrm{D}$ confining geometries. In Figs. 11 and 12 , the equilibrium partition coefficients $K_{0}$ are shown for a linear SAW polymer of $N=10^{3}$, a linear RW polymer of $N$ $=10^{3}$, a symmetric three-arm RW star polymer of arm length $N=10^{3}$, a symmetric four-arm RW star polymer of arm length $N=10^{3}$, and a sphere with a square channel confining geometry of $d \times d$ cross section and a $d \times d \times d$ cubic box confining geometry, respectively. Again, it is clear that among $R_{g}, R_{g} g^{-1 / 3}, R_{h}$, and $R_{s}$, the best universality in the partitioning behavior is provided by the use of our new size parameter $R_{s}$. In the universal partitioning regime, $K_{0}$ depends only on $R_{s} / d$. For a square channel for $d \times d$ cross section, we have

$$
K_{0, \text { square channel }}(d)=\left(1-2 R_{s} / d\right)^{2} \text {, }
$$

and for a $d \times d \times d$ cubic box confining geometry, we have
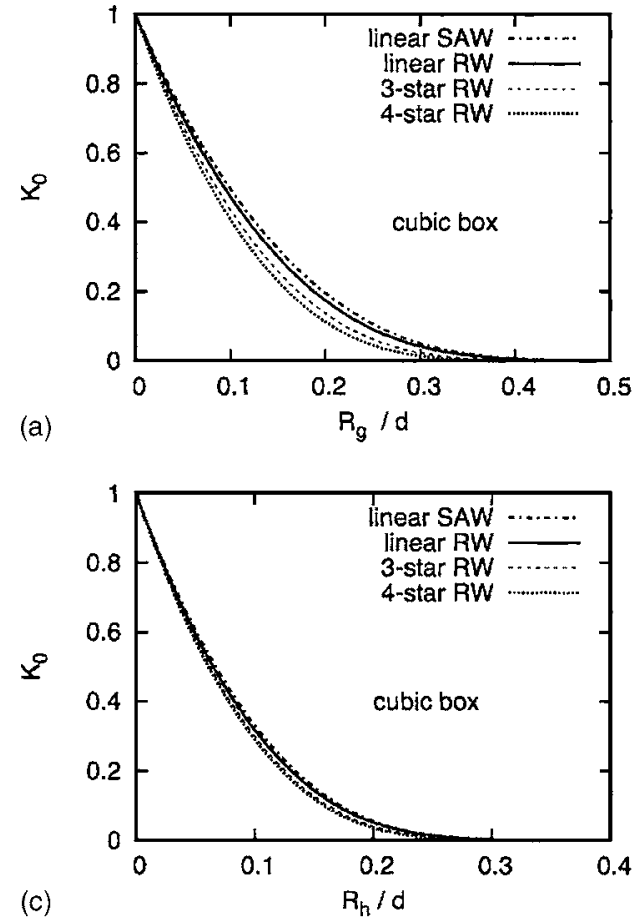
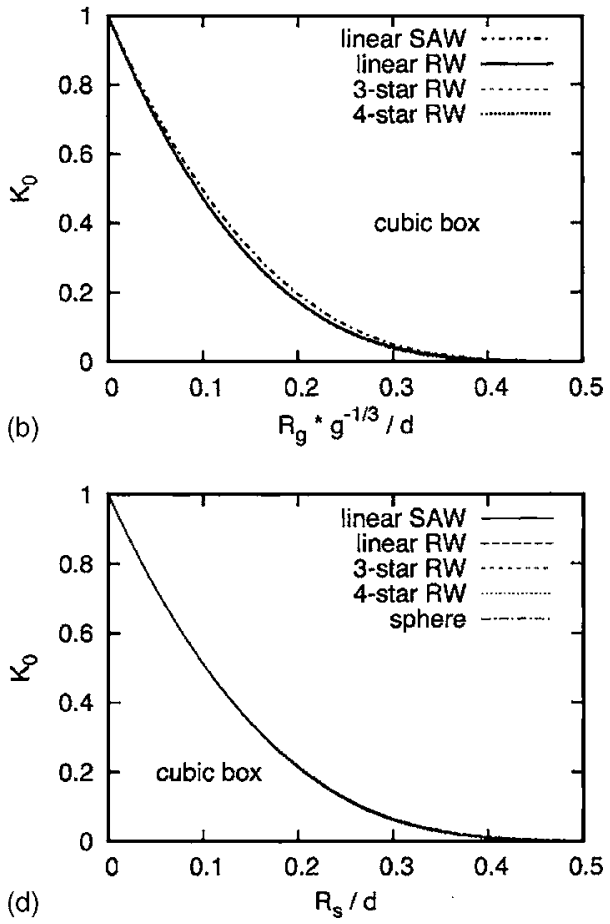

FIG. 12. Equilibrium partition coefficients $K_{0}$ are shown (a) as a function of $R_{g} / d$, (b) as a function of $R_{g} g^{-1 / 3} / d$, (c) as a function of $R_{h} / d$, and (d) as a function of $R_{s} / d$ for (from top to bottom) a linear SAW polymer of $N$ $=10^{3}$, a linear RW polymer of $N=10^{3}$, a symmetric three-arm RW star polymer of arm length $N=10^{3}$, and a symmetric four-arm RW star polymer of arm length $N=10^{3}$ with a cubic box confining geometry of size $d \times d \times d$. In subfigure (d), $K_{0}$ for a sphere is also shown. 


$$
K_{0, \text { cubic box }}(d)=\left(1-2 R_{S} / d\right)^{3} .
$$

Equations (52) and (53) are obtained from Eqs. (29) and (51). One may realize that Eqs. (29), (52), and (53) are essentially the equilibrium partitioning curves of a sphere of radius $R=R_{S}$ with a slit, a square channel, and a cubic box, respectively. Strictly speaking, the equilibrium partitioning curves for flexible polymers with channel and box confining geometries deviate from the universal partitioning behavior at approximately the same $R_{s} / d$ value as with a slit confining geometry. The reason that better universality appears in Figs. 11(d) and 12(d) is because, for the same $R_{s} / d$ value, the corresponding $K_{0}$ value decreases as the confinement dimension increases. When $K_{0}$ for a cubic box confining geometry is plotted as a function of $R_{s} / d$ (Fig. 12), one can barely see the difference among the different molecules studied.

Although it remains interesting to check the universal behavior in a plot of $K_{0}$ as a function of $R_{s} / d$ for confining geometries with curved surfaces (such as a cylindrical pore), it seems fair to conclude from Figs. 11 and 12 that the steric exclusion radius $R_{s}$, which is a geometric size measure, is the determining molecular size parameter that characterizes the equilibrium partitioning behavior of polymers with confining geometries. This result may indicate that polymers in SEC are separated based on their steric exclusion radii.

\section{CONCLUSIONS}

We have developed a new method to compute the equilibrium partition coefficient of macromolecules between bulk and confining geometries in the dilute solution limit. The computation method, called the confinement analysis from bulk structures (CABS), allows the computation of equilibrium partition coefficients as a function of confinement size on the basis of a single sampling of the configuration space of the macromolecule in bulk. Superior in computational speed to previous simulation studies, CABS is capable of handling slit, square channel, and cubic box confining geometries. Our focus was mainly on calculating $K_{0}$. However, we note here that the CABS method can also be extended to compute the equilibrium concentration profile inside a confining geometry and to study the conformational properties of confined macromolecules etc. The extension is straightforward, and will be presented in forthcoming publications.

CABS also leads to a new geometric molecular size parameter called the steric exclusion radius $R_{s}$. An explicit definition of $R_{s}$ is provided to facilitate the computation of $R_{s}$ by exploring the configuration space of a macromolecule during molecular simulations, and we have also obtained $R_{S}$ analytically for a number of rigid objects and flexible polymers. Distinct from conventional molecular size parameters such as the root-mean-square radius of gyration, the steric exclusion radius depends only on the chain segments that are located on the molecular surface.

We suggest that $R_{s}$ may be a more relevant molecular length scale for characterization of spatial confinement effects on macromolecules. In particular, results for the equilibrium partition coefficients as a function of the ratio of $R_{s}$ to the confinement size show a unique curve in the weak confinement regime. This result may indicate that the sepa- ration of macromolecules in SEC is based on the steric exclusion radius. Experimentally, Grubisic et al. ${ }^{34}$ showed that SEC separates polymers on the basis of hydrodynamic volume $V_{h}=M[\eta] / 6^{3 / 2} .^{51}$ They demonstrated that data plotted in the form of $\log ([\eta] M)$ versus elution volume nearly fell on a single curve for a wide range of different polymers. Such an excellent correlation between the hydrodynamic volume and elution volume is important in practice, but it is somewhat controversial to the thermodynamic separation principle generally accepted in theoretical studies since $V_{h}$ is a size based on dynamic quantities. ${ }^{35,52}$ Like other types of chromatography, SEC is typically run under low flow rate conditions where hydrodynamic factors should have little effect on the molecular partitioning. ${ }^{14,35}$ In the present work, we have demonstrated that the steric exclusion radius is the determining molecular size parameter that characterizes the equilibrium partitioning behavior, and this result may indicate that the steric exclusion radius and the hydrodynamic volume may be strongly correlated.

Note added in proof: We have recently discovered that the steric exclusion radius $R_{S}$ defined in Eq. (30) is in fact one-half the projection of the unconfined polymer molecule onto a line as discussed in Ref. 54. However, the concept does not seem to have been used in current textbooks on polymer physics ${ }^{2-4,38,42,53}$ and in a recent review article on confined polymer solutions. ${ }^{5}$

\section{ACKNOWLEDGMENTS}

Y.W. acknowledges support by the Danish Research Council for Technology and Production Sciences Grant No. 26-04-0074. G.H.P. acknowledges financial support from the Danish National Research Foundation via a grant to MEMPHYS-Center for Biomembrane Physics. Simulations were performed at the Danish Center for Scientific Computing at the Technical University of Denmark.

${ }^{1}$ E. F. Casassa, J. Polym. Sci., Part B: Polym. Lett. 5, 773 (1967).

${ }^{2}$ P. G. de Gennes, Scaling Concepts in Polymer Physics (Cornell University Press, Ithaca, 1979).

${ }^{3}$ M. Doi and S. F. Edwards, The Theory of Polymer Dynamics (Oxford University Press, New York, 1986).

${ }^{4}$ I. Teraoka, Polymer Solutions: An Introduction to Physical Properties (Wiley, New York, 2002).

${ }^{5}$ I. Teraoka, Prog. Polym. Sci. 21, 89 (1996).

${ }^{6}$ A. T. Clark and M. Lal, J. Chem. Soc., Faraday Trans. 2 77, 981 (1981).

${ }^{7}$ J. Ennis and B. Jönsson, J. Phys. Chem. B 103, 2248 (1999).

${ }^{8}$ Z. Chen and F. A. Escobedo, Macromolecules 34, 8802 (2001).

${ }^{9}$ P. Cifra and T. Bleha, Macromol. Theory Simul. 9, 555 (2000).

${ }^{10}$ T. Bleha and P. Cifra, Polymer 44, 3745 (2003).

${ }^{11}$ R. M. Jendrejack, D. C. Schwartz, J. J. de Pablo, and M. D. Graham, J. Chem. Phys. 120, 2513 (2004).

${ }^{12}$ J. P. Hernández-Ortiz, H. Ma, J. J. de Pablo, and M. D. Graham, Phys. Fluids 18, 123101 (2006).

${ }^{13}$ D. Stein, F. H. J. van der Heyden, W. J. A. Koopmans, and C. Dekker, Proc. Natl. Acad. Sci. U.S.A. 103, 15853 (2006).

${ }^{14}$ J. C. Giddings, E. Kucera, C. P. Russell, and M. N. Myers, J. Phys. Chem. 72, 4397 (1968).

${ }^{15}$ E. F. Casassa and Y. Tagami, Macromolecules 2, 14 (1969).

${ }^{16}$ I. Teraoka, Macromolecules 37, 6632 (2004).

${ }^{17}$ M. G. Davidson, U. W. Suter, and W. M. Deen, Macromolecules 20, 1141 (1987).

${ }^{18}$ M. Daoud and P. G. de Gennes, J. Phys. (Paris) 38, 85 (1977).

${ }^{19}$ Y. Wang and I. Teraoka, Macromolecules 30, 8473 (1997).

${ }^{20}$ E. Raphael and P. Pincus, J. Phys. II 2, 1341 (1992). 
${ }^{21}$ J. Dayantis and J. Sturm, Polymer 26, 1631 (1985).

${ }^{22}$ P. Cifra, T. Bleha, and A. Romanov, Polymer 29, 1664 (1988).

${ }^{23}$ T. Bleha, P. Cifra, and F. E. Karasz, Polymer 31, 1321 (1990).

${ }^{24}$ A. Yethiraj and C. K. Hall, Mol. Phys. 73, 503 (1991).

${ }^{25}$ W. Ji and Y. Wang, J. Chem. Phys. 121, 3905 (2004).

${ }^{26}$ P. Cifra and T. Bleha, Polymer 41, 1003 (2000).

${ }^{27}$ P. Cifra and T. Bleha, Macromolecules 34, 605 (2001).

${ }^{28}$ Y. Wang, I. Teraoka, and P. Cifra, Macromolecules 34, 127 (2001).

${ }^{29}$ P. Cifra and I. Teraoka, Polymer 43, 2409 (2002).

${ }^{30}$ Y. Wang, Q. Lin, P. Cifra, and I. Teraoka, Colloids Surf., A 206, 305 (2002).

${ }^{31}$ I. Teraoka, P. Cifra, and Y. Wang, Colloids Surf., A 206, 299 (2002).

${ }^{32}$ W. Radke, Macromol. Theory Simul. 10, 668 (2001).

${ }^{33}$ W. Radke, J. Chromatogr., A 1028, 211 (2004).

${ }^{34}$ Z. Grubisic, P. Rempp, and H. Benoit, J. Polym. Sci., Part B: Polym. Lett. 5, 753 (1967).

${ }^{35}$ T. Sun, R. R. Chance, W. W. Graessley, and D. J. Lohse, Macromolecules 37, 4304 (2004).

${ }^{36}$ J. Klein and M. Grüneberg, Macromolecules 14, 1411 (1981).

${ }^{37}$ B. H. Zimm and W. H. Stockmayer, J. Chem. Phys. 17, 1301 (1949).

${ }^{38}$ M. Doi, Introduction to Polymer Dynamics (Oxford University Press, New York, 1996).

${ }^{39}$ D. A. McQuarrie, Statistical Mechanics (University Science Books, Sausalito, CA, 2000)

${ }^{40}$ I. Teraoka, P. Cifra, and Y. Wang, Macromolecules 34, 7121 (2001).

${ }^{41}$ I. Teraoka, P. Cifra, and Y. Wang, Colloids Surf., A 206, 299 (2002).

${ }^{42}$ R. B. Bird, C. F. Curtiss, R. C. Armstrong, and O. Hassager, Dynamics of
Polymeric Liquids: Kinetic Theory (Wiley, New York, 1986), Vol. 2.

${ }^{43}$ S. Asakura and F. Oosawa, J. Polym. Sci. 33, 183 (1958).

${ }^{44}$ A. Vrij, Pure Appl. Chem. 48, 471 (1976).

${ }^{45}$ P. G. Bolhuis, A. A. Louis, J. P. Hansen, and E. J. Meijer, J. Chem. Phys. 114, 4296 (2001).

${ }^{46}$ A. A. Louis, P. G. Bolhuis, E. J. Meijer, and J. P. Hansen, J. Chem. Phys. 117, 1893 (2002).

${ }^{47}$ H. Tobita and S. Saito, Macromol. Theory Simul. 8, 513 (1999).

${ }^{48}$ T. Kennedy, J. Stat. Phys. 106, 407 (2002).

${ }^{49}$ D. P. Landau and K. Binder, A Guide to Monte Carlo Simulations in Statistical Physics (Cambridge University Press, Cambridge, England, 2000).

${ }^{50}$ B. Dünweg, D. Reith, M. Steinhauser, and K. Kremer, J. Chem. Phys. 117, 914 (2002).

${ }^{51}$ T. Konishi, T. Yoshizaki, and H. Yamakawa, Macromolecules 24, 5614 (1991).

${ }^{52}$ B. S. Farmer, K. Terao, and J. W. Mays, Int. J. Polym. Anal. Charact. 11, 3 (2006).

${ }^{53}$ M. Rubinstein and R. Colby, Polymer Physics (Oxford University Press, New York, 2003).

${ }^{54}$ H. E. Daniels, Proc. Cambridge Philos. Soc. 37, 244 (1941). H. Kuhn, Helv. Chim. Acta 31, 1677 (1948). J. J. Weidmann, H. Kuhn, and W. Kuhn, J. de Chemie Physique et de Physico-Chemie Biologique 50, 226 (1953). M. E. van Kreveld, J. Polym. Sci., Polym. Phys. Ed. 13, 2253 (1975). E. F. Casassa, Macromolecules 9, 182 (1976). E. F. Casassa, J. Appl. Polym. Sci.: Appl. Polym. Symp. 72, 151 (1985). 